\title{
Perfil dos pacientes com transplante hepático devido à insuficiência hepática aguda atendidos no Hospital Universitário Walter Cantídio
}

\section{Profile of patients with liver transplant due to acute liver failure attended the University Hospital Walter Cantídio}

Fellype Donato Chaves ${ }^{1}$. José Milton de Castro Lima ${ }^{2}$. José Huygens Parente Garcia ${ }^{3}$. Maria Izabel Jovino Silva $^{4}$. José Renan Miranda Cavalcante Filho 5 .

1 Residência Médica em Gastroenterologia, Hospital Universitário Walter Cantídio (HUWC), Fortaleza, Ceará, Brasil. 2 Doutor em Gastroenterologia, Professor associado pela Faculdade de Medicina, Universidade Federal do Ceará (UFC), Fortaleza, Ceará, Brasil. 3 Doutor em Cirurgia, Professor Titular pela Faculdade de Medicina, Universidade Federal do Ceará (UFC), Fortaleza, Ceará, Brasil. 4 Médica, Universidade de Fortaleza (UNIFOR), Fortaleza, Ceará, Brasil. 5 Acadêmico de Medicina, Universidade Federal do Ceará (UFC), Fortaleza, Ceará, Brasil.

\section{RESUMO}

Objetivo: Avaliar o perfil dos pacientes submetidos a transplante hepático em decorrência de insuficiência hepática aguda (IHA) atendidos no Hospital Universitário Walter Cantídio/UFC. Metodologia: Trata-se de estudo retrospectivo, longitudinal, observacional, no qual foram selecionados os pacientes submetidos a transplante hepático no período de janeiro de 2003 a julho de 2015, em decorrência de insuficiência hepática aguda. O coeficiente $\rho$ (Rô) de Spearman foi elencado para cálculo estatístico das correlações estudadas neste trabalho. Resultados: O sexo feminino correspondeu a 90,5\%, dos casos. A média de idade desses pacientes foi de 30,2 anos. Quanto à distribuição regional dos doentes acompanhados $66,6 \%$ dos indivíduos eram procedentes do Ceará, enquanto $33,4 \%$ residiam em outros estados. Na correlação entre a idade pré-transplante de 14 dos pacientes e a sobrevida por 1000 dias, obteve-se um coeficiente de correção de Spearman igual -0,004, não nos sugerindo que houve pior prognóstico para os pacientes mais velhos, quando estes desenvolveram IHA. Conclusão: A insuficiência hepática aguda, no espaço amostral do HUWC/UFC, é uma doença de adultos jovens, que acomete mais mulheres do que homens; em sua maioria pardos e negros, residentes predominantemente no interior do estado do Ceará.

Palavras-chave: Falência hepática aguda. Transplante de fígado. Encefalopatia hepática.

\section{ABSTRACT}

Objective: To evaluate the profile of patients undergoing liver transplantation as a result of acute hepatic insufficiency at the Walter Cantídio University Hospital / UFC. Methodology: This is a retrospective, longitudinal, observational, scientific study in which patients undergoing liver transplantation were selected from January 2003 to July 2015 as a result of acute liver failure (ALF). The Spearman coefficient $\rho$ (Rô) was used for the statistical calculation of the correlations studied in this study. Results: The female sex corresponded to $90.5 \%$ of the cases. The mean age of these patients was 30.2 years. Regarding the regional distribution of the patients, $66.6 \%$ of the individuals were from Ceará, while $33.4 \%$ were residing in other states. The correlation between the pretransplantation age of 14 patients and the survival time per 1000 days showed a Spearman's Rô of -0.004, which does not suggest a worse prognosis for the older patients when they developed ALF. Conclusion: From the data demonstrated, acute liver failure in the HUWC/UFC sample space is a disease of young adults, which affects more women than men; mostly brown and black people, living in Ceará - predominantly in the interior areas of the state.

Keywords: Liver failure, acute. Liver transplantation. Hepatic encephalopathy.

Autor correspondente: José Milton de Castro Lima, Rua Augusto Jaime Benevides, 900, Guararapes, Fortaleza, Ceará. CEP: 60810-331. Telefone: +55 85 99982-4376. E-mail: jmcl.lima@gmail.com

Conflito de interesses: Não há qualquer conflito de interesses por parte de qualquer um dos autores.

Recebido em: 06 Abr 2017; Revisado em: 24 Set 2017; Aceito em: 24 Set 2017. 


\section{INTRODUÇÃO}

A insuficiência hepática aguda (IHA) é definida como uma lesão hepatocelular associada à falência funcional do fígado, que implica em aumento da concentração sérica de amônia, encefalopatia, disfunção osmótica plasmática-tecidual, distúrbios de coagulação e comprometimento imunológico. ${ }^{1}$ Tal patologia tem prognóstico reservado quando não corrigida, preferencialmente, por um transplante hepático $(\mathrm{TxH})$ e necessita de investigação meticulosa para melhor enfrentamento.

A IHA apresenta uma alta morbidade e mortalidade. A maioria dos estudos sugerem que menos de $15 \%$ dos pacientes sobrevivem sem o transplante de fígado, apesar das melhorias na gestão de cuidados intensivos. O TxH melhorou significativamente o prognóstico dos pacientes com IHF. ${ }^{2}$

As taxas de mortalidade permanecem altas em razão da IHF. No entanto, reduziram com o decorrer dos anos, devido ao maior conhecimento sobre a patologia, terapias médicas mais agressivas, aprimoramento dos cuidados e advento do TxH. ${ }^{3}$

As causas da IHA variam grandemente entre os países. Nos EUA, em 1960, as mais frequentes eram a hepatite A e B. Atualmente, a hepatite B constitui apenas $7 \%$, enquanto a toxicidade pelo paracetamol representa em torno de $50 \%$ de todos os casos. ${ }^{4}$

A identificação da causa da IHA é importantíssima porque influencia o prognóstico e o manejo terapêutico da doença. Cerca de $17 \%$ dos casos permanecem indeterminados e este grupo provavelmente inclui pacientes com hepatites por vírus não-A-E, e possivelmente, metabólitos não reconhecidos ou doenças genéticas, especialmente na população pediátrica. ${ }^{5}$

A apresentação clínica vai depender da severidade e da etiologia do dano hepático. Sintomas inespecíficos como náuseas, vômitos e desconforto abdominal poderão ser a apresentação inicial dos pacientes com IHA. Os resultados dos testes de função hepática e coagulação indicarão a severidade da agressão ao fígado. Praticamente todos os sistemas são acometidos direta ou indiretamente nos casos graves. Podem evoluir com apresentação de encefalopatia, coagulopatia, alteração cardiovascular e nefrometabólica. ${ }^{4}$

A única intervenção terapêutica de benefício comprovado para pacientes com IHA é o transplante hepático de emergência. O prognóstico da IHA varia muito, dependendo da etiologia e de uma série de outros fatores. Com uma taxa de mortalidade superior a $80 \%$ sem o transplante de fígado, é de extrema importância o reconhecimento e o diagnóstico da IHA precocemente. ${ }^{1}$

Em 2002, foi realizado o primeiro TxH no estado do Ceará, no Hospital Universitário Walter Cantídio (HUWC/ UFC), determinando uma sobrevida de mais de dois anos pós-transplante. ${ }^{5}$ No ano de 2012, a associação brasileira de transplante de órgãos (ABTO) classificou o Ceará como um centro de referência nacional de transplante, com mais de
700 TxH já realizados e uma taxa de 18,9 doadores de fígado por milhão da população. ${ }^{6}$ Tendo em vista os poucos estudos com esta enfermidade, o presente estudo propõe-se a avaliar o perfil dos pacientes submetidos a TxH em decorrência de IHA atendidos no HUWC/UFC.

\section{MATERIAL E MÉTODOS}

Trata-se de um estudo retrospectivo, observacional, longitudinal, no qual foram selecionados os pacientes submetidos a TxH no período de janeiro de 2003 a julho de 2015, em decorrência de IHA. Os dados foram obtidos de registros disponíveis em prontuários digitais ou impressos.

Os critérios de exclusão foram: pacientes cujos prontuários encontravam-se avariados ou inacessíveis após duas tentativas de recuperação no serviço de registro do HUWC em dias diferentes.

Os resultados da análise sistematizada dos dados dos prontuários resgatados foram processados e avaliados através do software SPSS ${ }^{\circledR}$ (IBM) e posteriormente exportados para o Excel $^{\circledR}$ (Microsoft) no qual a construção de gráficos foi empreendida.

O coeficiente $\rho$ (Rô) de Spearman foi elencado para cálculo estatístico das correlações. A maioria das associações encontradas foram do tipo linear.

As variáveis dinâmicas estudadas foram índice de massa corpórea, de creatinina, Model for End-stage Liver Disease (MELD) em dois momentos antecedendo o TxH. As variáveis estáticas avaliadas foram: idade antecedendo o transplante hepático, sexo e grau de encefalopatia hepática. Essas variáveis, em sua maioria, foram testadas conjuntamente a desfechos relacionados à sobrevida de 60 a 1000 dias pós-transplante ortotópico de fígado.

O presente estudo foi devidamente submetido e aprovado pelo Comitê de Ética do Hospital Universitário Walter Cantidio, com o CAAE: 57209615.6.0000.5045.

\section{RESULTADOS}

Foram encontrados inicialmente 34 registros de pacientes com IHA que foram admitidos no HUWC e submetidos, em sua grande maioria, a TxH.

Contudo, em razão de inconformidades nos prontuários, falhas de preenchimento, ausência de exames laboratoriais, apenas em 21 registros pôde-se aplicar o instrumento de avaliação objetiva proposto no estudo.

A etiologia da IHA, em poucos casos, pôde ser inferida pela avaliação anatomopatológica. Os motivos foram a ausência de registro dos laudos em prontuário, ou mesmo por laconismo não objetivo na descrição dos achados nas peças cirúrgicas pós-transplante. 
A distribuição por gênero foi de $9,5 \%$ de pacientes do sexo masculino e $90,5 \%$ do sexo feminino (Gráfico 1), e média de idade de 30,2 anos (Desvio padrão DP $=15,34$ anos).

Com a correlação entre a idade pré-transplante de 14 dos pacientes e a sobrevida por 1000 dias, demonstrou-se um Rô de Spearman de $-0,004(\mathrm{p}=0,988)$, não sugerindo-nos que houve pior prognóstico para os pacientes mais velhos, quando estes desenvolveram IHA (Gráfico 2). Quanto à distribuição regional dos doentes acompanhados $66,6 \%$ dos indivíduos eram procedentes do Ceará, enquanto $33,4 \%$ residiam fora deste estado.

Gráfico 1. Distribuição percentual do sexo dos pacientes internados no HUWC por insuficiência hepática aguda.

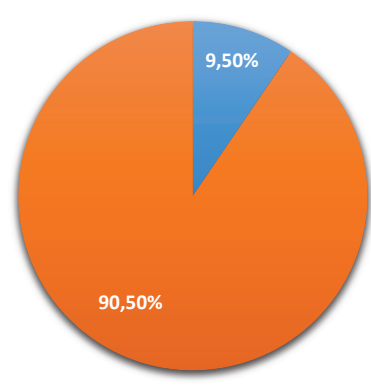

HOMENS MULHERES

Gráfico 2. Correlação entre a idade pré-transplante e a sobrevida, em dias, pós-transplante hepático.

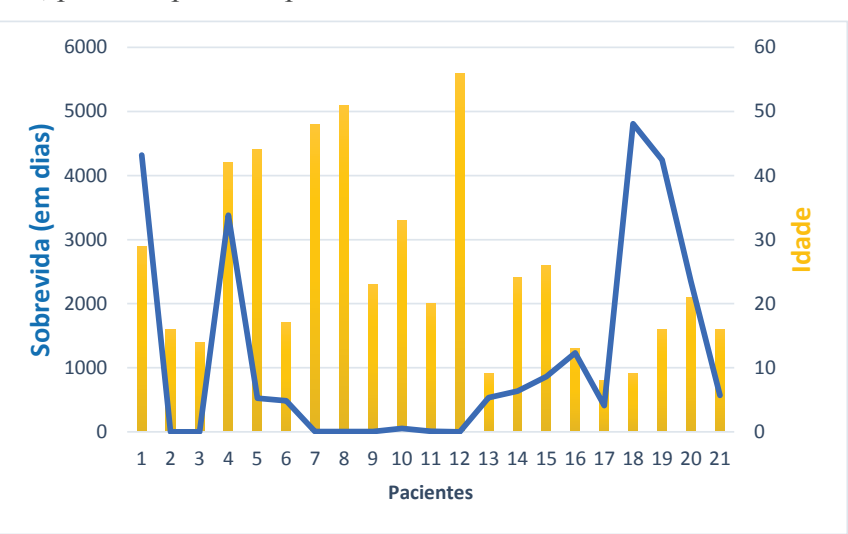

A avaliação étnica demonstrou que cerca de 50\% eram negros/ pardos. A renda familiar variou de menos de um, até três salários mínimos. Recebiam até um salário mínimo 26,7\% dos indivíduos; entre dois e três salários $26,7 \%$ e três salários mínimos ou mais $6,7 \%$. Percebeu-se que $40 \%$ não possuíam registros de suas rendas familiares em seus prontuários. Em relação à escolaridade, $66,6 \%$ eram alfabetizados. A análise do estado civil revelou que $66,7 \%$ eram solteiros; $23,8 \%$ eram casados e 4,8\% eram divorciados. Em $4,8 \%$ não foi possível identificar o estado civil pré-transplante. Encontrou-se como comorbidades mais frequentes o uso crônico de substâncias/medicações hepatotóxicas, a hipertensão arterial e o etilismo prévio.

Quanto à distribuição etiológica das insuficiências hepáticas agudas, 26,6\% eram de origem criptogênica; as hepatites A e as hepatotoxicidades por medicamentos corresponderam, cada uma, a $20 \%$ dos casos. A hemocromatose, a reação adversa a ervas medicinais, a associação de medicamentos com bebida alcoólica, a doença de Wilson e o uso de suplementos alimentares foram responsáveis por $6,7 \%$ da incidência, cada (Tabela 1).

Tabela 1. Distribuição da etiologia das IHAs em pacientes submetidos a transplante hepático por insuficiência hepática aguda. HUWC-UFC, 2003-2015.

\begin{tabular}{lll}
\hline Etiologia & $\begin{array}{l}\text { Valor } \\
\text { absoluto }\end{array}$ & $\begin{array}{l}\text { Valor } \\
\text { percentual }\end{array}$ \\
\hline Anticoncepcional injetável & 1 & $(6,7)$ \\
Criptogênica & 4 & $(26,6)$ \\
Doença de Wilson & 1 & $(6,7)$ \\
Droga (Metildopa) & 1 & $(6,7)$ \\
Ervas Medicinais & 1 & $(6,7)$ \\
Hemocromatose & 1 & $(6,7)$ \\
Hepatite A & 3 & $(20)$ \\
Libação Alcoólica Medicamentosa & 1 & $(6,7)$ \\
Medicamentosa (fenitoína+paracetamol) & 1 & $(6,7)$ \\
Suplemento alimentar & 1 & $(6,7)$ \\
\hline
\end{tabular}

Em 15 pacientes que foram submetidos a TxH pôde-se avaliar laudos anatomopatológicos dos seus explantes. Em 11 deles, a etiologia encontrada nas peças não coincidiu com as avaliações clínicas pré-transplantes. Nos três casos em que houve congruência entre a hipótese clínica e os achados histopatológicos, a etiologia por reação adversa a medicamentos foi a imputada. Houve um caso em que não foi possível definir a causa base da hepatite fulminante através da análise histopatológica.

A distribuição percentual dos pacientes nessa avaliação, segundo a classificação da velocidade de instalação da IHA proposta por $\mathrm{O}^{\prime} \mathrm{Grady}{ }^{7}$ foi de: $20 \%$ de casos de IHA hiperaguda; $33,3 \%$ de IHA agudas e $46,7 \%$ de IHA subagudas (Tabela 2, Gráfico 3).

Quando se avaliou a influência do grau de encefalopatia hepática pré-transplante, segundo os critérios de West-Haven, ${ }^{8}$ sobre a sobrevida em 1000 dias após o procedimento, foi encontrado um Rô de Spearman de 0,031 ( $\mathrm{p}=0,92)$. Devido ao baixo poder da amostra, com esses resultados não é possível afirmar a existência de correlação entre a elevação do grau de comprometimento neurológico e a sobrevida pós-transplante (Gráfico 4).

Tabela 2. Evolução da IHA x Sobrevida em pacientes submetidos a transplante hepático por insuficiência hepática aguda. HUWC-UFC, 2003-2015.

\begin{tabular}{llll}
\hline & & \multicolumn{2}{c}{ Sobrevida } \\
\hline \multirow{4}{*}{ Evolução } & & $<2$ meses & $>2$ meses \\
& Subaguda & 3 & 6 \\
& Aguda & 4 & 5 \\
& Hiperaguda & 1 & 1 \\
\hline
\end{tabular}


Gráfico 3. Distribuição das médias de sobrevida dos pacientes com insuficiência hepática aguda, conforme a classificação das insuficiências hepáticas agudas de O'Grady J.G. et al (1989).

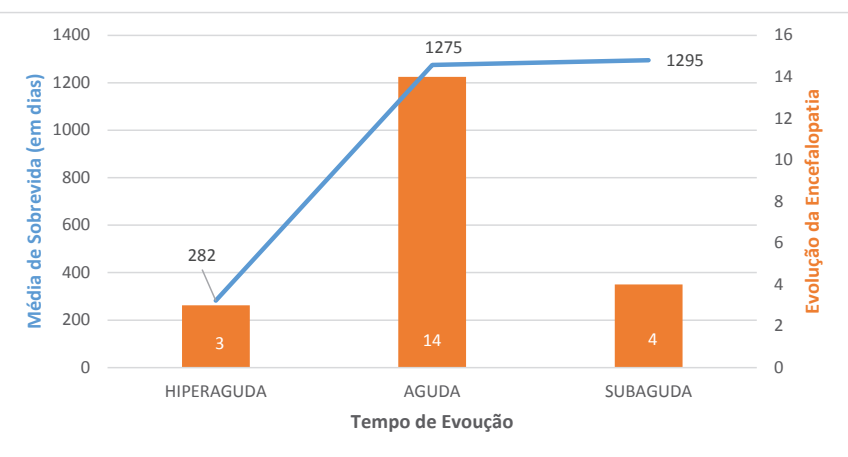

Gráfico 4. Correlação entre os graus de encefalopatia, conforme a escala de West-Haven, e a sobrevida em dias, após o transplante hepático.

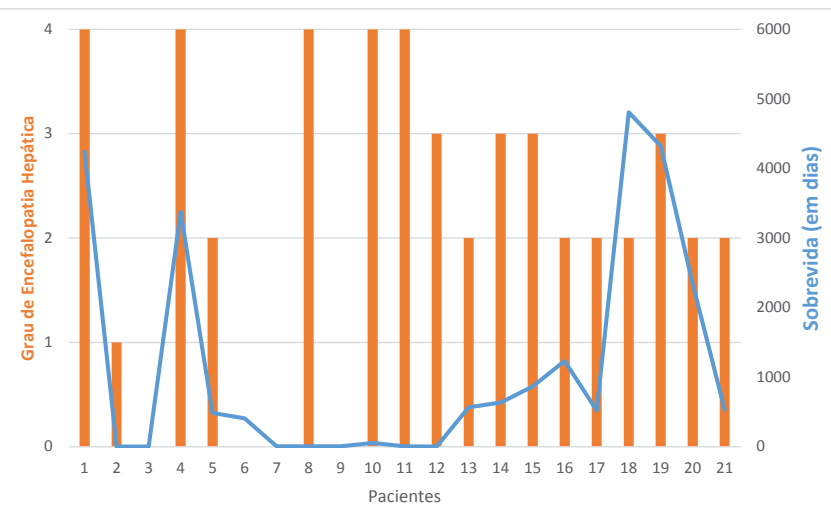

Em relação às repercussões os dados do estudo evidenciaram que $33,3 \%$ dos pacientes evoluíram com insuficiência renal superposta à insuficiência hepática aguda, necessitando de hemodiálise intermitente. Dentre todos, $66,7 \%$ não precisaram de terapia substitutiva renal. O uso de circulação extracorpórea foi necessário em $6,6 \%$ dos transplantes.

No pós-transplante, $73,3 \%$ dos doentes evoluíram com infecção. Do total de pacientes, 53,3\% desenvolveram sepse e $13,3 \%$ apresentaram choque séptico. Do total de pacientes, $26,6 \%$ necessitaram de drogas vasoativas (noradrenalina; vasopressina) no período pós-operatório, destes, $6,6 \%$ precisaram de octreotide.

Quanto à necessidade de ventilação mecânica, 46,6\% demandaram intubação orotraqueal. Houve necessidade de ventilação não-invasiva em $13,3 \%$ dos pacientes no seu pós-operatório.

A média dos índices de massa corpórea (IMC) pré-transplantes foi de 21,5035 ( $\mathrm{DP}=4,2301)$. Ressalta-se que a correlação entre IMC e sobrevida pós-transplante, calculada pelo Rô de Spearman como sendo -0,39; sugere pior prognóstico para os pacientes com maiores IMCs pré-transplante, contudo, sem atingir significância estatística (Gráfico 5, Tabela 3).
Tabela 3. Coeficientes de Correlação (Rô) de Spearman.

\begin{tabular}{lllllll}
\hline & & & & & & IMC pré- \\
& & MeldR1 & Idde2015 & Peso & Altura & transplante \\
\hline Sobrevida & $\mathrm{r}$ & 0,051 & $-0,206$ & $-0,05$ & $-0,149$ & $-0,039$ \\
& $\mathrm{p}$ & 0,858 & 0,384 & 0,838 & 0,541 & 0,875 \\
\hline
\end{tabular}

Gráfico 5. Correlação entre as faixas de índice de massa corpórea e a sobrevida pós-transplante hepático.

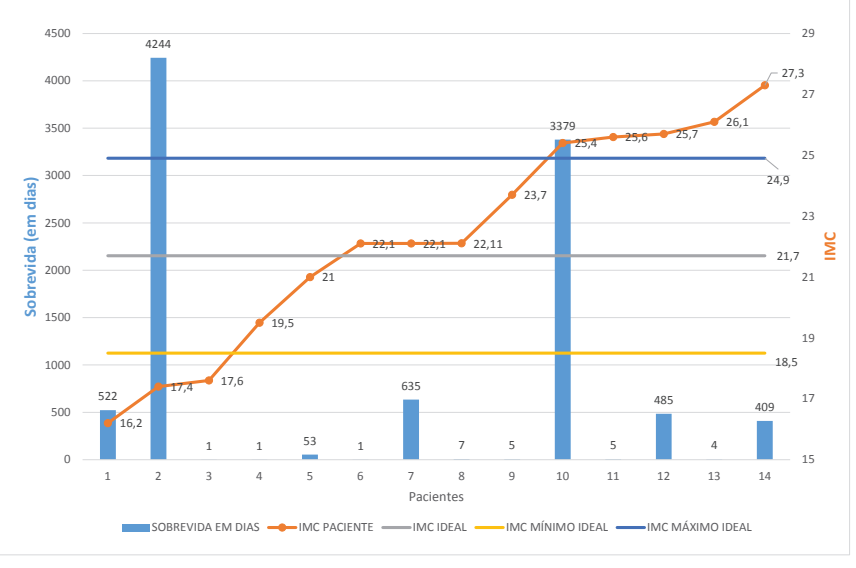

Segundo renomado estudo, ${ }^{9}$ o escore MELD pré-transplante hepático correlaciona-se com precisão ao risco de morte caso não seja realizado o procedimento salvador para o doente com IHA. Assim sendo, neste trabalho testou-se a correlação do MELD dos pacientes antes do TxH e a sobrevida por pelo menos 1000 dias depois. Encontrou-se um Rô de Spearman de $-0,409$; com $p=0,275$. A correlação que se pôde fazer é de piores resultados de sobrevida pós-transplante ortotópico de fígado para os indivíduos que atingiram maiores escores MELD no HUWC.

Em extensa série de casos, comenta-se que para pacientes com elevados MELD pré-transplante, o uso de drogas vasoativas constitui, junto à necessidade maior de transfusão de hemoderivados, importante fator de morbidade. ${ }^{10}$

Diante de tal relevância foi estabelecida uma correlação entre a influência da necessidade de drogas vasoativas pré-transplante hepático sobre a sobrevida 1000 dias após o procedimento. Foi evidenciado um Rô de Spearman de -0,657 com p=0,011, ou seja, com significância estatística.

Também foi avaliada a distribuição da taxa de mortalidade dos pacientes transplantados por insuficiência hepática aguda correlacionando-a com o tempo, em dias, após o procedimento. $60 \%$ das mortes ocorreram após 120 dias, 35\% em menos de 30 dias e $5 \%$ entre 31 e 60 dias (Tabela 4). Em 6,66\% dos casos de pacientes com insuficiência hepática aguda de nossa série houve morte no pós-operatório imediato. Em outros $6,66 \%$ o óbito sobreveio antes da realização do transplante hepático prontamente indicado. 
Tabela 4. Taxa de mortalidade de acordo com o tempo pós-transplante hepático por IAH. HUWC-UFC, 2003-2015.

\begin{tabular}{ll}
\hline Dias pós-transplante & Porcentagem de Mortalidade \\
\hline Menor que 30 dias & $35 \%$ \\
Entre 31 e 60 dias & $5 \%$ \\
Entre 61 e 120 dias & $0 \%$ \\
Maior que 120 dias & $60 \%$ \\
\hline
\end{tabular}

\section{DISCUSSÃO}

$\mathrm{Na}$ análise empreendida no HUWC, os pacientes tiveram idade variando de 9 a 56 anos, com média de 30,2 anos (desvio padrão $\mathrm{DP}=15,34$ anos). Constatando-se então uma possível incidência aumentada de IHA em populações mais jovens; ou ainda subnotificação dos casos em pacientes mais velhos.

Quanto à distribuição entre gêneros das insuficiências hepáticas agudas na casuística do HUWC, é patente e expressivo a predominância de casos sobre o sexo feminino; cuja prevalência em nossa amostra foi de 90,5\% em contraste com os 9,5\% de homens com IHA, à semelhança de outros estudos. ${ }^{2,11}$

No presente estudo obteve-se um coeficiente de correlação (Rô) de Spearman entre a idade antecedendo o transplante hepático e a sobrevida em 1000 dias após o procedimento, igual a -0,004. Isto nos sugere prognóstico similar em pacientes de mais idade quando comparados a pacientes mais jovens. ${ }^{11}$

Alguns dos pacientes possuíam comorbidades, principalmente hipertensão arterial, uso crônico de medicações hepatotóxicas e etilismo crônico. É bem descrito que pacientes com hipertensão, diabetes ou insuficiência renal pré-transplante, possuem elevado risco de morte após o procedimento. ${ }^{10}$

As etiologias das IHAs observadas entre os casos do HUWC distribuíram-se como criptogênica em $26,6 \%$ dos casos. As intoxicações por medicamentos corresponderam a 26,7\%. O papel das hepatites virais, principalmente a hepatite A, mostrou-se elevado na casuística cearense, sendo responsável por $20 \%$ dos registros. Outras patologias, tais como a doença de Wilson e a hemocromatose foram responsáveis cada uma por $6,7 \%$ dos registros. O uso concomitante de medicações hepatotóxicas com álcool, as reações adversas às plantas medicinais e o uso de suplementos alimentares foram responsáveis por mais $6,6 \%$, cada. Tal distribuição assemelha-se com outras já relatadas na literatura. ${ }^{12}$ Houve preponderância das etiologias virais e idiopáticas como principais implicadas na necessidade de transplante hepático, também nas insuficiências hepáticas agudas.

Os pacientes avaliados por nosso estudo tiveram suas IHAs classificadas de acordo com a evolução clínica segundo os critérios utilizados por renomado estudo. ${ }^{7}$ Os casos de insuficiência hepática hiperaguda constituíram 20\%, $33,3 \%$ dos doentes apresentaram insuficiências hepáticas classificáveis como agudas e 46,7\% tiveram IHAs subagudas.
A preponderância de casos de insuficiência hepática subaguda e aguda em nossa população estudada é distinta, por exemplo, dos resultados de estudo multicêntrico nos Estados Unidos. ${ }^{3}$ Tal estudo apresentou predomínio de IHA aguda e hiperaguda, principalmente relacionadas à ingestão de paracetamol. Nesse referido trabalho, o achado das IHAs subagudas esteve mais associado ao uso crônico de medicações hepatotóxicas ou a reações idiossincrásicas a elas.

Quando analisado o desfecho sobrevida em 1000 dias após o procedimento, os achados do estudo corroboram com dados relatados na literatura, evidenciando melhor prognóstico para os pacientes com evoluções hiperagudas nas IHAs por toxicidade ao paracetamol ou outras drogas, bem como o desfecho pior para as hepatites soronegativas e reações a medicamentos que se associaram à instalação subaguda do quadro de falência hepática aguda. ${ }^{13}$

Na série de casos descrita, 33,3\% dos doentes apresentaram insuficiência renal no período anterior ou pouco após a realização do TxH. Em nossa realidade clínica, 66,6\% dos doentes não precisaram de terapia renal substitutiva e o uso de circulação extracorpórea foi necessário em 6,6\% dos transplantes de fígado. Há menção ao pior prognóstico pós-transplantes nas patologias hepáticas graves quando a condição de base impõe injúria aos rins concomitantemente. ${ }^{10,14}$

É descrita na literatura maior vulnerabilidade dos doentes acometidos por IHA a infecções, sendo uma característica peculiar à sua síndrome clínica. ${ }^{9} \mathrm{Na}$ casuística do HUWC, 73,3\% evoluíram com infecção no perioperatório. Em torno de 53,3\% desenvolveram sepse e 13,3\% apresentaram choque séptico.

O uso de drogas vasoativas (noradrenalina, vasopressina) fezse necessário a $26,6 \%$ dos pacientes no período pós-operatório. O octreotide foi demandado por $6,6 \%$ dos pacientes transplantados por IHA. A ventilação mecânica invasiva foi necessária em 46,6\% dos doentes, e a ventilação mecânica não invasiva foi empreendida em $13,3 \%$ dos pacientes durante o pós-operatório.

Nos registros de pacientes em que se pôde encontrar os dados para o cálculo do índice de massa corpórea (IMC) - 19 doentes -, calculou-se uma média de 21,5035 ( $\mathrm{DP}=4,23018)$. A partir desses dados, conforme os indícios já relatados em estudo, ${ }^{15}$ traçamos uma correlação entre o IMC pré-transplante e a sobrevida após o procedimento. No cálculo do Rô de Spearman, encontrou-se um coeficiente de correlação de $-0,039$ com $p=0,875$.

Apesar de não ter sido originalmente projetado para avaliação de doentes com IHA, o escore MELD correlaciona-se com considerável precisão ao risco de morte em pacientes não submetidos a transplante hepático, inclusive os secundários a IHA. ${ }^{9}$ Em nosso estudo, a correlação entre os valores de MELD pré-transplante e a sobrevida 1000 dias após o procedimento foi analisada matematicamente pelo cálculo do Rô de Spearman, cujo valor foi de $-0,409$, com $p=0,275$. Ainda que o limiar de significância estatística não tenha sido atingido neste teste, e a sua interpretação tenha de ser feita com modéstia e prudência, 
sugere-se que, também para os pacientes com insuficiência hepática aguda, maiores MELD antecedendo o transplante hepático estão, em algum grau, associados a menores taxas de sobrevida nos três primeiros anos do enxerto.

Pelos dados demonstrados, percebe-se que a insuficiência hepática aguda, no espaço amostral do HUWC/UFC, é uma doença de adultos jovens, que acomete mais mulheres do que

\section{REFERÊNCIAS}

1. Sass DA, Shakil AO. Fulminant hepatic failure. Liver Transpl. 2005;11:594-604.

2. Viana CF, Rocha TD, Cavalcante FP, Valença JT Jr, Coelho GR, Garcia JH. Liver transplantation for acute liver failure - a 5 years experience. Arq Gastroenterol. 2008;45(3):192-4.

3. Ostapowicz G, Fontana RJ, Schiodt FV, Larson A, Davern TJ, Han $\mathrm{SH}$, et al. Results of a prospective study of acute liver failure at 17 tertiary care centers in the United States. Ann Intern Med. 2002;137(12):947-54.

4. Fonseca OC Neto. Fulminant hepatic failure: etiology, management and indications for liver transplantation. Arq Bras Cir Dig. 2008;21(4):201-4.

5. Garcia JH, Vasconcelos JB, Brasil IR, Costa PE, Vieira RP, Moraes MO. Liver transplantation: preliminary results. Rev Col Bras Cir. 2005;32(2):100-3.

6. Associação Brasileira de Transplante de Órgãos (ABTO). Registro brasileiro de transplantes. ABTO [Internet]. 2012 [acesso em: 22 abr 2017];18(4):9. Disponível em: http://www.abto.org.br/abtov03/ Upload/file/RBT/2012/rbt2012-parciall.pdf

7. O'Grady JG, Schalm SW, Williams R. Acute liver failure: redefining the syndromes. Lancet. 1993;342(8866):273-5.

8. Trey C, Davidson CS. The management of fulminant hepatic homens, em sua maioria pardos e negros, residentes no Ceará - predominantemente no interior do Estado.

Para melhor compreender a influência dos diversos fatores retrospectivamente elencados nesse trabalho, estudos de cortes prospectivos, com melhor rigor na compilação de dados e atento seguimento dos pacientes por períodos preestabelecidos devem ser produzidos.

failure. In: Popper H, Shaffner F, editores. Progress in liver diseases. 3. ed. New York: Grune \& Stratton; 1970. p. 282-98.

9. Castaldo ET, Chari RS. Liver transplantation for acute hepatic failure. HPB (Oxford). 2006;8(1):29-34.

10. Coêlho GR. Fatores associados com diminuição da sobrevida em 555 transplantes de fígado realizados na Universidade Federal do Ceará [Tese de doutorado]. Fortaleza: Faculdade de Medicina; 2013. $174 \mathrm{p}$.

11. Lee MW, Squires RH Jr, Nyberg SL, Doo E, Hoofnagle JH. Acute liver failure: summary of a workshop. Hepatology. 2008;47(4):1401-15.

12. Garcia JH, Mesquita DF, Coelho GR, Feitosa BA Neto, Nogueira EA, Silva AC Filho, et al. Results from a liver transplant center in northeastern Brazil that performed more than 100 transplants in 2011. Transplant Proc. 2014;46:1803-6.

13. Bernal W, Wendon J. Liver transplantation in adults with acute liver failure. J Hepatol. 2004;40(2):192-7.

14. Anand AC, Nightingale P, Neuberger JM. Early indicators of prognosis in fulminant hepatic failure: an assessment of the King's criteria. J Hepatol. 1997;26(1):62-8.

15. Rutherford A, Davern T, Hay JE, Murray NG, Hassanein T, Lee $\mathrm{WM}$, et al. Influence of high body mass index on outcome in acute liver failure. Cli Gastroenterol Hepatol. 2006;4(12):1544-9.

\section{Como citar:}

Chaves FD, Lima JM, Garcia JH, Silva MI, Cavalcante JR Filho. Perfil dos pacientes com transplante hepático devido à insuficiência hepática aguda atendidos no Hospital Universitário Walter Cantídio. Rev Med UFC. 2018 abr-jun;58(2):41-46. 